\title{
Conceptions of Vibrational Signatures Based on Chiral/Helical Functionalized Helicenes Nanostructures: Analyzed of Normal and Identical Modes
}

\author{
Simplice Koudjina ${ }^{1,3, *}$, Wilfried G. Kanhounnon ${ }^{1}$, Gaston A. Kpotin ${ }^{1}$, Nobel Kouakou N'Guessan ${ }^{2}$ \\ and Guy Y. S. Atohoun ${ }^{1}$ \\ ${ }^{1}$ Laboratoire de Chimie Théorique et de Spectroscopie, Moléculaire (LACTHESMO), Université d'Abomey- \\ Calavi (UAC), 03 BP 3409 Cotonou-Bénin \\ ${ }^{2}$ Laboratoire de Thermodynamique et Physico-Chimie du Milieu (LTPCM), UFR-SFA, Université Nangui \\ Abrogoua (UNA), 02 B.P. 801 Abidjan 02 - Côte d'Ivoire \\ ${ }^{3}$ Ecole Nationale Supérieure des Biosciences et Biotechnologies Appliquées (ENSBBA), Université Nationale \\ des Sciences, Technologies, Ingénierie et Mathématiques (UNSTIM), BP 2282 Goho Abomey-Bénin
}

\begin{abstract}
Optoelectronics properties as helical molecular fingerprints have been investigated on a set of Helicenes molecules, which form a particular class of compounds and exhibit both $\pi$-electron delocalization and chiral properties. In this paper, we investigate the IR and Raman signatures of four representative Helicenes: Hexahilicene (Hexa-Helicene), tetrathia-[7]-helicene (Helicene-4S), and its pyrrole (Helicene$4 \mathrm{~N}$ ) and furan analogs (Helicene-4O), under the visible wavelength of $532 \mathrm{~nm}$. Correctly, the impact of the method of calculation on these signatures has been pointed out. The simulation of the IR and Raman signatures involves two different steps: the evaluation of the vibrational frequencies and normal modes and the calculation of the Cartesian derivatives of

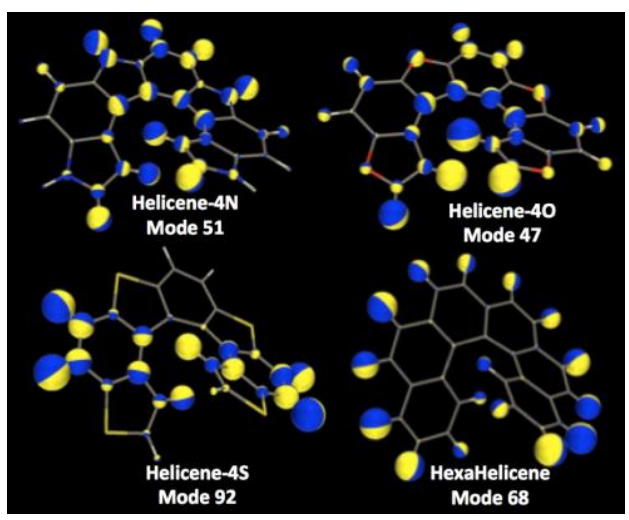
electric properties. While most of the time, all the quantities are evaluated with a single method, we believe that this should not be the case since both steps have not the same requirements in terms of computational methods. Density functional theory has been then used with different exchange-correlation functional and Coupled Perturbed Time-Dependent Hartree-Fock (CP-TDHF) for the electric properties investigations. It comes out of the results that B3LYP, B3P86, and PBE0, reproduces better experimental spectra. The impact of the electron correlation as view one the $\mathrm{XC}$ functional on the evaluation of the Cartesian derivatives of the electric properties were found to be somewhat limited. Overall, the most crucial point is to have an accurate description of the normal vibrational modes via the choice of appropriate XC functionals, which describe the experiment results.
\end{abstract}

Keywords: Vibrational signatures; Helicenes; XC; IR; Raman; CP-TDHF; Chiral/Helical.

\section{Introduction}

Helicene molecules, which are made of ortho-fused rings, have unique properties combining the electron delocalization and helicity ${ }^{1-4}$. Therefore, they exhibit chiroptical properties, such as optical rotation ${ }^{5-7}$ and specific electronic circular dichroism bands ${ }^{8,9}$. In addition, their electronic responses, chirality also leads to remarkable vibrational properties, which can be probed using Vibrational Circular Dichroism (VCD) and Vibrational Raman Optical Activity
(VROA) spectroscopies ${ }^{10,11}$. For instance, the VCD and VROA vibrational signatures (together with their non-chiral IR and Raman spectroscopies counterparts) of Hexa- and Hepta-Helicenes have been investigated both experimentally and theoretically ${ }^{12,13}$.

In the present study, we want to address the impact of the method on the simulation of IR and Raman signatures (while their optically active counterparts should follow the same requirements and will be investigated in future work). 
The simulation of such spectra requires the evaluation of the vibrational frequencies and normal modes as well as the calculation of Cartesian derivatives of the electric dipole moment ${ }^{14}(\partial \mu / \partial \mathrm{R})$ and the electric polarizability ${ }^{15,16}(\partial \alpha / \partial \mathrm{R})$. While, most of the time, all these quantities are calculated in one step, the requirement in terms of methods may not be the same for the vibrational frequencies, normal modes and the evaluation of the Cartesian derivatives of the two electrical properties derivatives $(\partial \mu / \partial \mathrm{R}$ and $\partial \alpha / \partial \mathrm{R}){ }^{17,18}$. These electric properties have been observed on IR and Raman spectra of each Helicenes molecules, which could be seen afterward by a geometry deformation and regular modes perturbations. Therefore, we propose to analyze first the impact of the method on the frequencies and normal vibrational modes and, in a second step, the impact on the Cartesian derivatives of electric properties. The paper is organized as in five sections as follow: After the introduction, a review on the quantities needed to simulate the IR and Raman spectra in one hand, and in the other hand the different tools useful to analyze the impact of the method on these quantities are presented in Section 2. In section 3 , the overall methodology is explained. The obtained results are presented and discussed in Section 4. We end up with some conclusions (Section 5) and acknowledgments.

\section{Computational Theoretical Aspects}

In a first step, the geometric structures of four Helicenes investigated here have been fully optimized under the caster of Density Functional Theory (DFT) with nine exchange-correlation (XC) functionals: B3P86, BP86, LC-BLYP, M06, M06-2X, PBE0, $\omega B 97 X, \omega B 97 X-D$, and the reference B3LYP together with three different basis sets: the standard 6-31G* split-valence basis set of Pople et al. ${ }^{19}$. The correlation-consistent double and triple-zeta (cc-pVDZ, cc-pVTZ) basis sets of Dunning et al. ${ }^{20}$. Thus, the vibrational frequencies and normal modes were determined by adopting the analytical CoupledPerturbed Kohn-Sham (CPKS) ${ }^{21}$ procedure using the same method as for the optimization of geometry. The hybrid B3LYP exchange-correlation functional has been selected as a reference, owing to its recognized performance for calculating vibrational frequencies ${ }^{22}$. In order to take into account the systematic error due to both missing electron correlation and anharmonicity effects, a multiplicative factor of 0.96 is employed to scale the B3LYP vibrational frequencies ${ }^{23,24}$. For the other XC functionals, a homemade factor is used, as described in section 2 . In a third step, the Cartesian derivatives of the dipole moment (to describe IR intensity) and of the polarizability (to describe Raman intensity) were evaluated with the Coupled Perturbed TimeDependent Hartree-Fock (CP-TDHF) scheme for frequencies calculations, using the aug-cc-pVDZ ${ }^{20}$ bases set as our reference method.
These calculations enabled us to analyze the impact of the method (XC functional and basis set) on the normal vibrational modes and their IR and Raman signatures while keeping the same way for evaluation of the properties derivatives. Also, we have investigated the impact of the $\mathrm{XC}$ functional on the properties derivatives for the B3LYP/cc-pVTZ optimized structures. Six of nine $\mathrm{XC}$ functionals (B3LYP, B3P86, BP86, M06, M06-2X, and PBE0) have been used for that while in addition to the augcc-pVDZ basis set. Five more basis set have also been employed: $6-31 G^{* 19}, 6-31++G^{* 19}$, the rDPS:3-21G (reduced diffuse polarization function and shell augmented) basis set introduced by Zuber and Hug ${ }^{25}$ which consists of the $3-21++\mathrm{G}$ basis set, supplemented by a set of $p$ diffuse functions (of exponent 0.2) on the hydrogen atoms. The correlation-consistent double and triple-zeta (cc-pVDZ, cc-pVTZ) basis sets of Dunning ${ }^{20}$, and the extensive Sadlej-pVTZ basis set ${ }^{26}$, which was developed specifically for computations of electric properties, including dipole polarizabilities ${ }^{27}$.

The derivatives are evaluated for the Cartesian displacements and were transformed afterward into geometrical derivatives concerning the normal vibrational coordinates calculated using CPKS procedure. All the calculations were performed using the Gaussian/09-D01 ${ }^{28}$ software package.

We used a visible wavelength of $532 \mathrm{~nm}$, as in the experiment condition, to simulate the Raman spectra. The Maxwell-Boltzmann $\left(1 /\left[1-e^{\left(-\hbar \Delta \omega_{p} /\left(k_{b} T\right)\right)}\right] A\right)$ factor with $\mathrm{T}=298.15 \mathrm{~K}$ was used to account for the Temperature-dependence of the populations of the vibrational levels in the Raman spectra. In the spectra, each transition is represented by a Lorentzian function with FWHM of $10 \mathrm{~cm}^{-1}$, which is more or less what is obtained in the experimental spectra ${ }^{27}$.

\section{Vibrational Signatures of Normal Modes Process}

Some of IR and Raman spectra have been simulated at the doubled-harmonic approximation. In this approximation, the normal vibrational modes constitute a basis in which the mass-weighted Hessian matrix, $H_{i \alpha, j \beta}^{m}=\frac{1}{\sqrt{m_{i} m_{j}}}\left(\frac{\partial^{2} E}{\partial R_{i \alpha} \partial R_{j \beta}}\right)_{0}$, is diagonal ${ }^{29-31}$ :

$Q^{T} H^{m} Q=H^{q}$

The individual elements of $H^{q}$ are equal to the squares of the angular frequencies, $H_{p p}^{m}=\omega_{p}^{2}=$ $4 \pi^{2} v_{p}^{2}$, with $v_{p}$ being the $p$-th vibrational frequency. The $p$-th column of the unitary matrix $\boldsymbol{Q}$, denoted $\boldsymbol{Q}_{p}$, is the $p$-th normal mode in terms of mass-weighted Cartesian coordinates. The components of this mode in terms of Cartesian coordinates $\boldsymbol{Q}_{p}^{c}$ is then expressed as $Q_{i \alpha, p}^{c}=\left(\frac{1}{\sqrt{m_{i}}}\right) Q_{i \alpha, p}$. In the matrix terms, the indexes $i$ and $j$ stand for atomic nuclei while $\alpha$ and $\beta$ are used for Cartesian components $(x, y, z)$. 
Within the harmonic approximation, the IR and Raman intensities associated to the $p$-th vibrational normal mode respectively read ${ }^{32-34}$ :

$$
\begin{aligned}
& \int_{\text {band }_{p}} \epsilon(\bar{v}) d \bar{v}=I_{p}^{I R}=\frac{N_{A}}{12 c^{2} \epsilon_{0}} \sum_{\alpha}\left(\frac{\partial^{2} \mu_{\alpha}}{\partial Q_{p}^{2}}\right) \quad\left[m . m^{-1}\right] \\
& n_{d \sigma(\theta)_{S C P, p}}=\frac{1}{90}\left(\frac{\mu_{0}}{4 \pi}\right)^{2} \omega_{p}^{3} \omega_{0} \frac{\hbar}{2 \Delta \omega_{p}}\left[A . \alpha_{p}^{2}+B \cdot \beta_{p}^{2}\right] d \Omega\left[m^{2} / s r\right]
\end{aligned}
$$

Where $c$ is the speed of light in vacuum, $\omega_{0}=2 \pi v_{0}$ is the angular frequency of the laser beam, $\omega_{p}$ the angular frequency of the scattered light, $\Delta \omega_{p}$ corresponds to the vibrational transition associated with the normal coordinate $Q_{p}, \epsilon_{0}$ is the electric constant (formerly vacuum permittivity), $\hbar$ is the Planck constant divided by $2 \pi$, and $N_{A}$ is the Avogadro number.

$\sigma$ defines the rate at which photons are removed from the incident beam by scattering relative to the rate at which they cross a unit area perpendicular to the direction of propagation of the incident beam.

A, B are multiplicative factors that are a function of the scattering angle $\theta$. For instance, for the Raman polarized intensity $(\theta=\pi / 2, \perp$ Component), they amount to 90 , and 14 , respectively while for the Raman depolarized intensity $(\theta=\pi / 2$, $\|$ Component), they amount to 0 , and 12 respectively.

$\alpha_{p}^{2}, \beta_{p}^{2}$ in Eq. (2) are the two Raman invariants that require the evaluation of the first-order derivatives of three polarizability tensors $\left(\partial \alpha / \partial R_{i \alpha}\right)^{35}$ :

$\alpha_{p}^{2}=\frac{1}{9} \sum_{\mu, v}\left(\frac{\partial \alpha_{\mu \mu}}{\partial Q_{p}}\right)_{0}\left(\frac{\partial \alpha_{v v}}{\partial Q_{p}}\right)_{0}$

$\beta_{p}^{2}=\frac{1}{2} \sum_{\mu, v}\left[3\left(\frac{\partial \alpha_{\mu v}}{\partial Q_{p}}\right)_{0}\left(\frac{\partial \alpha_{\mu v}}{\partial Q_{p}}\right)_{0}-\left(\frac{\partial \alpha_{\mu \mu}}{\partial Q_{p}}\right)_{0}\left(\frac{\partial \alpha_{v v}}{\partial Q_{p}}\right)_{0}\right]$

In the above summations, the indices $\mu$, and $v$, are components of the electric fields $(x, y, z)$. The subscript 0 indicates that the properties are evaluated at the equilibrium geometry.

In this paper, the equilibrium geometry of Helicenes molecules is obtained with various exchange- correlation functionals and/or multiple atomic basis sets. In other to quantify the difference of geometry obtained by these different methods, the root means square deviation (RMSD) value between two structures is used:

$R M S D_{A, B}=\sqrt{\frac{\sum_{i}^{N}\left(R_{i x}^{A}-R_{i x}^{B}\right)^{2}+\left(R_{i y}^{A}-R_{i y}^{B}\right)^{2}+\left(R_{i z}^{A}-R_{i z}^{B}\right)^{2}}{N}}[\AA]$

Where the summation runs over all the $N$ atoms.

To rationalize the relationship between the normal modes obtained with various methods, we define the overlap matrix as the square of the scalar product between two standard ways expressed in the massweighted coordinates.

The overlap between the normal mode $\ell$ of method A and mode $p$ of methods B reads:

$O_{\ell, p}^{A, B}=\left\langle Q_{\ell}^{A} \mid Q_{p}^{B}\right\rangle^{2}=\left(\sum_{i \alpha}^{3 N} Q_{i \alpha, \ell}^{A} Q_{i \alpha, p}^{B}\right)^{2}$

Since the normal modes are normalized and form a complete basis set, one can easily show that sum of the overlap of a normal mode $\ell(\operatorname{method} \mathrm{A})$ with all the modes of method $B$ give a value of one:

$\left\langle Q_{\ell}^{A} \mid Q_{\ell}^{A}\right\rangle=1=\sum_{p}^{B}\left\langle Q_{\ell}^{A} \mid Q_{p}^{B}\right\rangle\left\langle Q_{p}^{B} \mid Q_{\ell}^{A}\right\rangle=\sum_{p}^{B} O_{\ell, p}^{A, B}$

Table 1 displays a part of this matrix when comparing the B3LYP/cc-pVTZ and B3P86/cc-pVTZ normal modes of Helicene-4N. To analyze even further this matrix, we introduce the concept of identical modes and non-equivalent modes. A normal mode is said to be identical between two methods when its overlap value is greater than or equal to a threshold value set at $\mathbf{0 . 9 5 0}$ throughout this work.

Table 1. Subblock of the overlap matrix between the B3LYP/cc-pVTZ (Lines) and B3P86/cc-pVTZ (Columns) normal modes of Helicene-4N in the $1200-1600 \mathrm{~cm}^{-1}$ wavenumber region. The bold values indicate major

\begin{tabular}{|c|c|c|c|c|c|c|c|}
\hline & & \multicolumn{6}{|c|}{ Freq/B3LYP } \\
\hline \multicolumn{2}{|c|}{ Freq/B3P86 } & 1217.197 & 1230.031 & 1242.918 & 1265.216 & 1265.662 & 1268.111 \\
\hline & & 70 & 71 & 72 & 73 & 74 & 75 \\
\hline 1224.750 & 70 & 0.9644 & 0.0000 & 0.0266 & 0.0000 & 0.0022 & 0.0000 \\
\hline 1230.179 & 71 & 0.0000 & 0.9951 & 0.0000 & 0.0000 & 0.0000 & 0.0000 \\
\hline 1246.042 & 72 & 0.0242 & 0.0000 & 0.9593 & 0.0000 & 0.0094 & 0.0000 \\
\hline 1271.469 & 73 & 0.0000 & 0.0000 & 0.0000 & 0.7339 & 0.0000 & 0.2491 \\
\hline 1272.220 & 74 & 0.0039 & 0.0000 & 0.0083 & 0.0000 & 0.9764 & 0.0000 \\
\hline 1278.491 & 75 & 0.0000 & 0.0000 & 0.0000 & 0.2519 & 0.0000 & 0.7338 \\
\hline
\end{tabular}
contributions (> 0.20). 
From six normal modes in Table 1, four of them (mode 70, 71, 72, and 74 for both methods) satisfied this definition and are therefore called identical modes. The last two modes (73 and 75) do not have a single homolog between the two methods and are consequently called non-equivalent modes and correspond to a combination of modes.

If we consider that a simple scalar factor $\mathrm{k}$ connects the elements of the mass-weighted Hessian matrix between two methods:

$$
\begin{aligned}
& H^{m, A}=k^{2} H^{m, B} \\
& \omega^{2, A}=k^{2} \omega^{2, B}
\end{aligned}
$$

The result would be that the two mass-weighted Hessian have the same set of eigenvectors and that their eigenvalues are related by the $k^{2}$ scalar factor. Most of the time, this situation is not real, and the relationship between the two mass-weighted Hessian is more complicated. However, for the identical modes, such a relationship is approximately correct, and it would be therefore interesting to do a linear correlation between the vibrational frequencies of those identical modes between two methods:

$\bar{v}^{A}=k \bar{v}^{B}$

The slope of the linear correlation $(k)$ is determined using a least-squares procedure that minimizes the following error ${ }^{23,24}$ :

$\Delta=\sum_{i}^{N_{\text {ind modes }}}\left(k \bar{v}_{i}^{B}-\bar{v}_{i}^{A}\right)^{2}$

Where the summation runs over all the identical modes. The slope is therefore read:

$k=\frac{\sum_{i}^{N_{\text {ind modes }}} \bar{v}_{i}^{A} \bar{v}_{i}^{B}}{\sum_{i}^{N_{\text {ind } \operatorname{modes}}\left(\bar{v}_{i}^{B}\right)^{2}}}$

Where $\bar{v}_{i}^{A}$ and $\bar{v}_{i}^{B}$ are the harmonic frequencies obtained with methods $\mathrm{A}$ and $\mathrm{B}$, respectively.

From this, one can define the root mean square deviation (RMSD) between the frequencies of method A (taken as reference) and the corrected frequencies of method B:

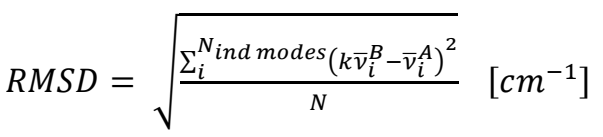

Using the overlap matrix and the RMSD ${ }^{36,37}$ between the frequencies of the identical modes, we can rationalize the changes in the normal modes between different methods. In this work, we used B3LYP/ccpVTZ as our reference method since it gives reliable geometries and normal modes of vibration. Meanwhile, to have more accurate vibrational frequencies, one needs to multiply the vibrational frequencies by a factor as tabulated by Scott and Radom ${ }^{23,24}$. For B3LYP, this factor amount to $\lambda^{B 3 L Y P}=0.96^{25}$. For a better comparison between our results obtained with the different methods and our reference B3LYP/cc-pVTZ results, we used the factors tabulated by Scott and Radom (which are obtained for a specific set of molecules) but we used the slope of the linear regressions between these methods and B3LYP/cc-pVTZ $\left(k_{B 3 L Y P / \text { Method }}\right)$ :

$\lambda^{\text {Method }}=\lambda^{B 3 L Y P} k_{B 3 L Y P / \text { Method }}$

These $\lambda^{\text {Method }}$ are, therefore, specific to each method and each molecule. Nevertheless, the same factor is used between different basis sets.

At last, to quantify the difference between the Infrared or Raman spectra obtained with two different methods, in the region of interest frequencies (200$1650 \mathrm{~cm}^{-1}$ ), the " $\mathrm{S}$ " quantify ${ }^{29-31}$ was calculated as the ratio between the intensity $I_{A}$ and $I_{B}$ overlap of these spectra and normalized on the square root of their self-overlap:

$S_{A, B}=\frac{\left\langle I_{A} \mid I_{B}\right\rangle}{\sqrt{\left\langle I_{A} \mid I_{A}\right\rangle\left\langle I_{B} \mid I_{B}\right\rangle}}$

Where $I_{A}$ is the overlap intensity in method A and $I_{B}$ the overlap intensity in methods $B$, respectively.

The overlap of the spectra is done in the frequency region of interest $\left(200-1650 \mathrm{~cm}^{-1}\right)$. The value of this quantity then amounts to 0 , or 1 , when the spectra are different or identical, respectively.

\section{Results and discussion}

\subsection{Applications on Helicenes Functionalized Nanostructures}

The geometric structures in the B3LYP/cc-pVTZ calculation method are optimized of four Helicenes (Helicene-4N, Helicene-4O, Helicene-4S, HexaHelicene), which are depicted in (Fig. 1).

(i) the reference Hexa-Helicene, which is made of six ortho-fused benzene rings,

(ii) Tetrathia-[7]-Helicene (Helicene-4S), which has recently attracted much interest owing to its facile synthesis and functionalization by donor and acceptor groups ${ }^{29-31}$ and

(iii) the analogous of Helicene-4S, where the four thiophene rings are replaced by furan or pyrrole rings (Helicene-4O and Helicene-4N) ${ }^{29-31}$.

Changing the nature of the heteroaromatic rings leads to the modification of electron delocalization and related molecular properties. 


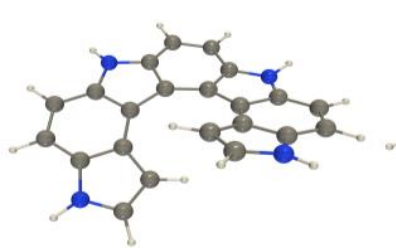

Helicene-4N

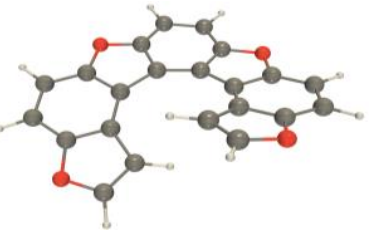

Helicene-40

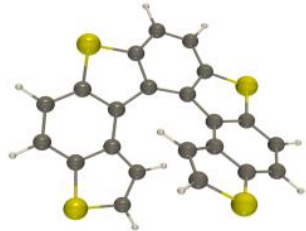

Helicene-4S

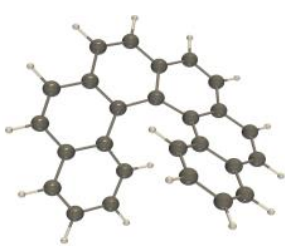

Hexa-Helicene

Figure 1. Sketch of the B3LYP/cc-pVTZ optimized geometrical structures of Helicene-4N, Helicene-4O, Helicene-4S and Hexa-Helicene, showed in profile view DrawMol homemade software package

\subsection{Effects of Exchange-Correlation Functionals} on the Vibrational Normal Modes

In this section, we analyze the impact of the $\mathrm{XC}$ functional on the normal vibrational modes. For this investigation, we have calculated the overlaps between the normal vibrational modes of the eight various functionals (B3P86, BP86, LC-BLYP, M06,
M06-2X, PBE0, $\omega$ B97X, $\omega B$ 97X-D) with cc-pVTZ basis set for B3LYP/cc-pVTZ basis set as a reference method for all calculations of Helicene-4N, Helicene4O, Helicene-4S, and Hexa-Helicene. The overlap matrices are analyzed in terms of identical modes and non-equivalent modes that are in Table 2.

Table 2. Numbers of Normal, Identical, Permuted, and Non-equivalent Modes between the variables functionals cc-pVTZ and B3LYP reference with cc-pVTZ basis set for Helicene-4N, Helicene-4O, Helicene-4S, and HexaHelicene. A normal mode is said to be identical between two methods when its overlap value is greater than or equal to a threshold value set at 0.950 .

\begin{tabular}{|c|c|c|c|c|c|c|c|c|}
\hline & \multicolumn{4}{|c|}{ Helicene-4N } & \multicolumn{4}{|c|}{ Helicene-40 } \\
\hline Functionals & $\begin{array}{l}\text { Normal } \\
\text { Modes }\end{array}$ & $\begin{array}{c}\text { Identical } \\
\text { Modes }\end{array}$ & $\begin{array}{l}\text { Permuted } \\
\text { Modes }\end{array}$ & $\begin{array}{l}\text { Non- } \\
\text { equivalent } \\
\text { Modes }\end{array}$ & $\begin{array}{l}\text { Normal } \\
\text { Modes }\end{array}$ & $\begin{array}{c}\text { Identical } \\
\text { Modes }\end{array}$ & $\begin{array}{c}\text { Permuted } \\
\text { Modes }\end{array}$ & $\begin{array}{l}\text { Non- } \\
\text { equivalent } \\
\text { Modes }\end{array}$ \\
\hline B3P86 & 114 & 92 & 7 & 22 & 102 & 90 & 4 & 12 \\
\hline BP86 & 114 & 89 & 7 & 25 & 102 & 80 & 4 & 22 \\
\hline LC-BLYP & 114 & 59 & 13 & 55 & 102 & 57 & 13 & 45 \\
\hline M06 & 114 & 78 & 14 & 36 & 102 & 76 & 2 & 26 \\
\hline M06-2X & 114 & 63 & 13 & 51 & 102 & 56 & 8 & 46 \\
\hline PBE0 & 114 & 83 & 8 & 31 & 102 & 81 & 5 & 21 \\
\hline$\omega B 97 X$ & 114 & 65 & 9 & 49 & 102 & 63 & 10 & 39 \\
\hline \multirow[t]{2}{*}{$\omega B 97 X-D$} & 114 & 67 & 11 & 47 & 102 & 68 & 6 & 34 \\
\hline & \multicolumn{4}{|c|}{ Helicene-4S } & \multicolumn{4}{|c|}{ Hexa-Helicene } \\
\hline Functionals & $\begin{array}{l}\text { Normal } \\
\text { Modes }\end{array}$ & $\begin{array}{c}\text { Identical } \\
\text { Modes }\end{array}$ & $\begin{array}{l}\text { Permuted } \\
\text { Modes }\end{array}$ & $\begin{array}{l}\text { Non- } \\
\text { equivalent } \\
\text { Modes }\end{array}$ & $\begin{array}{l}\text { Normal } \\
\text { Modes }\end{array}$ & $\begin{array}{c}\text { Identical } \\
\text { Modes }\end{array}$ & $\begin{array}{l}\text { Permuted } \\
\text { Modes }\end{array}$ & $\begin{array}{l}\text { Non- } \\
\text { equivalent } \\
\text { Modes }\end{array}$ \\
\hline B3P86 & 102 & 83 & 6 & 19 & 120 & 107 & 8 & 13 \\
\hline BP86 & 102 & 72 & 5 & 30 & 120 & 92 & 13 & 28 \\
\hline LC-BLYP & 102 & 61 & 3 & 41 & 120 & 64 & 9 & 56 \\
\hline M06 & 102 & 63 & 8 & 39 & 120 & 71 & 7 & 49 \\
\hline M06-2X & 102 & 57 & 4 & 45 & 120 & 69 & 9 & 51 \\
\hline PBE0 & 102 & 79 & 5 & 23 & 120 & 98 & 11 & 22 \\
\hline$\omega B 97 X$ & 102 & 67 & 5 & 35 & 120 & 69 & 11 & 51 \\
\hline$\omega B 97 X-D$ & 102 & 74 & 6 & 28 & 120 & 75 & 9 & 45 \\
\hline
\end{tabular}

We observe that B3P86, BP86, and PBE0 give normal modes that are closer to the reference ones with $81 \%$, $78 \%$ and $73 \%$ of identical modes for Helicene- $4 \mathrm{~N}$, respectively while these values are slightly larger for Helicene-4O with $88 \%, 78 \%$ and $79 \%$ of identical modes. For Hexa-Helicene, the same functionals give normal modes that are closer to the reference ones with $89 \%, 77 \%$, and $82 \%$ of identical modes, respectively.

However, for Helicen-4S, we found that B3P86, $\mathrm{PBE} 0$, and $\omega \mathrm{B} 97 \mathrm{X}-\mathrm{D} \mathrm{XC}$ functionals gave normal 
modes that are closer to the reference ones with $81 \%$, $71 \%$ and $73 \%$ of identical modes, respectively.

The effect of the XC functionals on the frequencies has been quantified by performing a linear regression between sets of vibrational frequencies in the $200-1650 \mathrm{~cm}^{-1}$ wavenumber region associated with the previously identified identical modes (Table 3 ).

Table 3. Linear regression (LR) between the functionals/cc-pVTZ frequencies and the B3LYP/cc-pVTZ frequencies for the identical modes in the range $200-1650 \mathrm{~cm}^{-1}$ for Helicene-4N, Helicene-4O, Helicene-4S, and Hexa-Helicene.

\begin{tabular}{|c|c|c|c|c|c|c|}
\hline \multirow[b]{2}{*}{ Functionals } & \multicolumn{3}{|c|}{ Helicene-4N } & \multicolumn{3}{|c|}{ Helicene-40 } \\
\hline & $\begin{array}{l}\text { Normal Modes } \\
\text { in LR }\end{array}$ & Slope of LR & $\operatorname{RMSD}\left(\mathrm{cm}^{-1}\right)$ & $\begin{array}{l}\text { Normal Modes } \\
\text { in LR }\end{array}$ & Slope of LR & $\operatorname{RMSD}\left(\mathrm{cm}^{-1}\right)$ \\
\hline B3P86 & 72 & 0.9932 & 5.12 & 74 & 0.9923 & 4.92 \\
\hline BP86 & 69 & 1.0321 & 5.97 & 64 & 1.0346 & 5.27 \\
\hline LC-BLYP & 39 & 0.9609 & 14.21 & 41 & 0.9525 & 10.05 \\
\hline M06 & 58 & 1.0005 & 6.96 & 60 & 0.9932 & 5.84 \\
\hline M06-2X & 47 & 0.9890 & 8.48 & 48 & 0.9837 & 8.93 \\
\hline PBE0 & 63 & 0.9881 & 6.89 & 65 & 0.9868 & 6.70 \\
\hline$\omega \mathrm{B} 97 \mathrm{X}$ & 45 & 0.9826 & 7.80 & 47 & 0.9777 & 10.16 \\
\hline \multirow[t]{2}{*}{$\omega B 97 X-D$} & 47 & 0.9832 & 5.86 & 52 & 0.9800 & 7.33 \\
\hline & \multicolumn{3}{|c|}{ Helicene-4S } & \multicolumn{3}{|c|}{ Hexa-Helicene } \\
\hline Functionals & $\begin{array}{c}\text { Normal } \\
\text { Modes in LR }\end{array}$ & Slope of LR & $\operatorname{RMSD}\left(\mathbf{c m}^{-1}\right)$ & $\begin{array}{l}\text { Normal Modes } \\
\text { in LR }\end{array}$ & Slope of LR & $\operatorname{RMSD}\left(\mathrm{cm}^{-1}\right)$ \\
\hline B3P86 & 65 & 0.9931 & 4.44 & 85 & 0.9952 & 5.61 \\
\hline BP86 & 58 & 1.0335 & 4.95 & 71 & 1.0349 & 4.77 \\
\hline LC-BLYP & 43 & 0.9450 & 9.54 & 40 & 0.9557 & 13.57 \\
\hline M06 & 50 & 0.9991 & 5.34 & 60 & 0.9964 & 6.79 \\
\hline M06-2X & 51 & 0.9848 & 6.50 & 59 & 0.9871 & 9.58 \\
\hline PBE0 & 61 & 0.9887 & 5.82 & 75 & 0.9925 & 7.30 \\
\hline$\omega B 97 X$ & 50 & 0.9718 & 7.05 & 52 & 0.9815 & 10.91 \\
\hline$\omega B 97 X-D$ & 59 & 0.9792 & 4.68 & 59 & 0.9853 & 7.01 \\
\hline
\end{tabular}

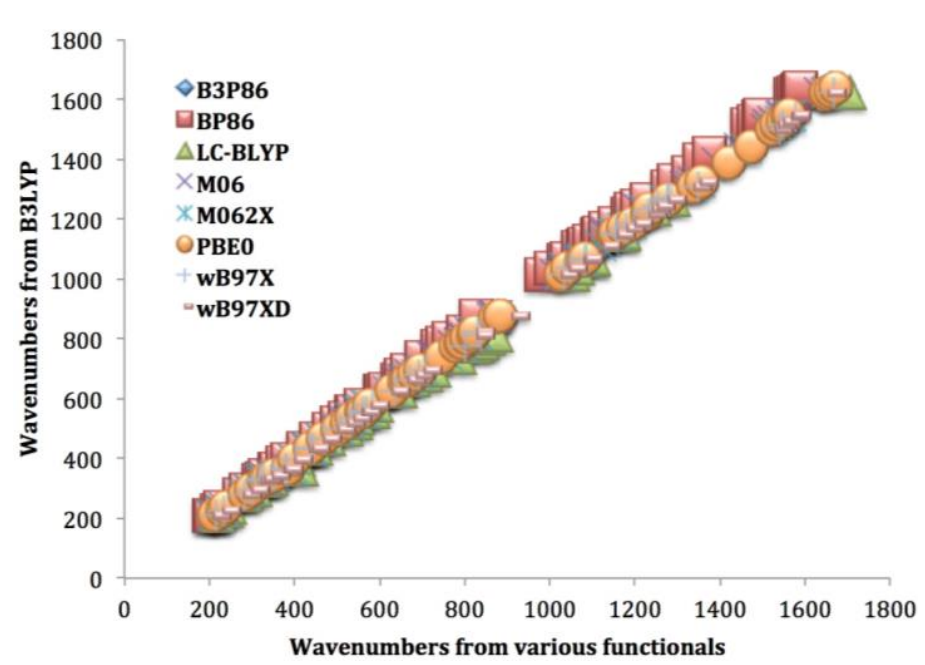

Figure 2. Correlation between the frequencies obtained with B3LYP and the frequencies obtained with the other selected XC-functionals

The lowest RSMD values are obtained for B3P86, BP86, M06, PBE0, and $\omega B$ 97X-D with values between 4.44 and $7.30 \mathrm{~cm}^{-1}$. From the different slopes, we can define the factors to correct the frequencies using Eq. (15). The different values for the four molecules and the different $\mathrm{XC}$ functionals are listed in Table 4. 
Table 4. Scaling factor for Helicene-4N, Helicene-4O, Helicene-4S, and Hexa-Helicene calculated as the B3LYP scaling (0.9600) times the slope of the linear regression between the frequencies of a given functional/cc-pVTZ and the frequencies obtained with B3LYP/cc-pVTZ for the identical modes in the range $200-1650 \mathrm{~cm}^{-1}$.

\begin{tabular}{|c|c|c|c|c|}
\hline \multirow{2}{*}{ Functionals } & \multicolumn{4}{|c|}{ Scaling Factor } \\
\cline { 2 - 5 } & Helicene-4N & Helicene-4O & Helicene-4S & Hexa-Helicene \\
\hline B3LYP & $\mathbf{0 . 9 6 0 0}$ & $\mathbf{0 . 9 6 0 0}$ & $\mathbf{0 . 9 6 0 0}$ & $\mathbf{0 . 9 6 0 0}$ \\
\hline B3P86 & 0.9534 & 0.9526 & 0.9534 & 0.9554 \\
\hline BP86 & 0.9908 & 0.9932 & 0.9922 & 0.9935 \\
\hline LC-BLYP & 0.9225 & 0.9144 & 0.9072 & 0.9175 \\
\hline M06 & 0.9605 & 0.9535 & 0.9591 & 0.9565 \\
\hline M06-2X & 0.9494 & 0.9444 & 0.9454 & 0.9476 \\
\hline PBE0 & 0.9486 & 0.9473 & 0.9492 & 0.9528 \\
\hline$\omega B 97 X$ & 0.9433 & 0.9386 & 0.9329 & 0.9422 \\
\hline$\omega$ B97X-D & 0.9439 & 0.9408 & 0.9400 & 0.9459 \\
\hline
\end{tabular}

Finally, we analyze the IR and Raman signatures when changing the $\mathrm{XC}$ functionals in the calculation of the normal modes. For that, the Cartesian derivatives of the dipole moment and the polarizability have been evaluated at the CP-TDHF/aug-cc-pVDZ level of theory and then projected on the normal modes that have just been analyzed. The frequencies of the normal modes have been corrected by the factors tabulated in Table 4.

The overlap ("S" values as defined by Equation (16)) between the IR and Raman spectra obtained with the normal modes calculated with different $\mathrm{XC}$ functionals and those obtained with the normal modes calculated with B3LYP functional. One should note that when taking the overlap between two spectra, the maximum intensity of the peaks might be slightly shifted between the methods, even after the correction on the frequencies. That is why a specific shift is allowed to correct even further the frequencies obtained with one method compared to the reference one. Therefore, a positive shift means that the frequencies are too high compare to the reference ones. In the calculation of the " $\mathrm{S}$ " values, the shift is tuned to a maximum of $\pm 50 \mathrm{~cm}^{-1}$ until a maximum " $\mathrm{S}$ " value is found between two spectra (Table 5).

Table 5. Overlap (Ov) and Shift $\left(\mathrm{cm}^{-1}\right)$, between the functionals/cc-pVTZ//CP-TDHF/aug-ccpVDZ spectra and the B3LYP/cc-pVTZ//CP-TDHF/aug-cc-pVDZ spectra in the range $200-1650 \mathrm{~cm}^{-1}$ for Helicene-4N, Helicene-4O, Helicene-4S, and Hexa-Helicene. The first method refers to the optimization of geometry and evaluation of the normal modes. In contrast, the second method (which CP-TDHF/aug-cc-pVDZ for all spectra) refers to the calculation of the property derivatives. The value of the IR displacement of $-28 \mathrm{~cm}^{-1}$ obtained with the LC-BLYP functional is overestimated, this may be due to the chemical shifts of the hydrogen $\mathrm{H}$ atoms attached to the $\mathrm{N}$ nitrogen in the Helicene-4N molecule. This is part of the reason why the LC-BLYP functionality is eliminated in the rest of the work for reading vibrational signatures.

\begin{tabular}{|c|c|c|c|c|c|c|c|c|}
\hline \multirow[b]{3}{*}{ Functionals } & \multicolumn{4}{|c|}{ Helicene-4N } & \multicolumn{4}{|c|}{ Helicene-4O } \\
\hline & \multicolumn{2}{|c|}{ IR } & \multicolumn{2}{|c|}{ Raman polarized } & \multicolumn{2}{|c|}{ IR } & \multicolumn{2}{|c|}{ Raman polarized } \\
\hline & $O v$ & Shift & $O v$ & Shift & $O v$ & Shift & $O v$ & Shift \\
\hline B3P86 & 0.8240 & 4.0 & 0.9605 & 10.0 & 0.9230 & -1.0 & 0.9320 & 9.0 \\
\hline BP86 & 0.8091 & 4.0 & 0.9469 & 2.0 & 0.8751 & -3.0 & 0.9767 & 4.0 \\
\hline LC-BLYP & 0.6556 & -28.0 & 0.8499 & 36.0 & 0.8106 & 4.0 & 0.9220 & 19.0 \\
\hline M06 & 0.7830 & 6.0 & 0.8873 & 21.0 & 0.8937 & -2.0 & 0.8791 & 11.0 \\
\hline M06-2X & 0.7462 & 2.0 & 0.8530 & 25.0 & 0.8199 & 3.0 & 0.9206 & 18.0 \\
\hline PBE0 & 0.7931 & 2.0 & 0.9415 & 14.0 & 0.8875 & 0.0 & 0.8974 & 12.0 \\
\hline$\omega \mathrm{B} 97 \mathrm{X}$ & 0.7316 & -1.0 & 0.8499 & 33.0 & 0.8088 & 9.0 & 0.9129 & 22.0 \\
\hline$\omega B 97 X-D$ & 0.8209 & 3.0 & 0.8532 & 22.0 & 0.8586 & 3.0 & 0.9325 & 14.0 \\
\hline
\end{tabular}




\begin{tabular}{|c|c|c|c|c|c|c|c|c|}
\hline & \multicolumn{4}{|c|}{ Helicene-4S } & \multicolumn{4}{c|}{ Hexa-Helicene } \\
\hline & \multicolumn{2}{|c|}{ IR } & \multicolumn{2}{|c|}{ Raman polarized } & \multicolumn{2}{|c|}{ IR } & \multicolumn{2}{c|}{ Raman polarized } \\
\hline Functionals & $\boldsymbol{O} \boldsymbol{v}$ & Shift & $\boldsymbol{O} \boldsymbol{v}$ & Shift & $\boldsymbol{O} \boldsymbol{v}$ & Shift & $\boldsymbol{O} \boldsymbol{v}$ & Shift \\
\hline B3P86 & 0.8425 & -1.0 & 0.8757 & 8.0 & 0.9919 & -3.0 & 0.9520 & 18.0 \\
\hline BP86 & 0.7175 & 0.0 & 0.8023 & 1.0 & 0.9827 & -4.0 & 0.9600 & 18.0 \\
\hline LC-BLYP & 0.7649 & -1.0 & 0.5622 & 24.0 & 0.9349 & -1.0 & 0.9204 & 11.0 \\
\hline M06 & 0.9015 & -2.0 & 0.8628 & 13.0 & 0.9828 & -5.0 & 0.9406 & 21.0 \\
\hline M06-2X & 0.8449 & 2.0 & 0.7469 & 7.0 & 0.9494 & 0.0 & 0.9502 & 13.0 \\
\hline PBE0 & 0.8127 & -2.0 & 0.8659 & 14.0 & 0.9866 & -4.0 & 0.9420 & 26.0 \\
\hline$\omega B 97 X$ & 0.8252 & 2.0 & 0.6223 & 3.0 & 0.9683 & -1.0 & 0.9367 & 19.0 \\
\hline$\omega B 97 X-D$ & 0.8760 & 2.0 & 0.7050 & 8.0 & 0.9860 & -7.0 & 0.9450 & 13.0 \\
\hline
\end{tabular}
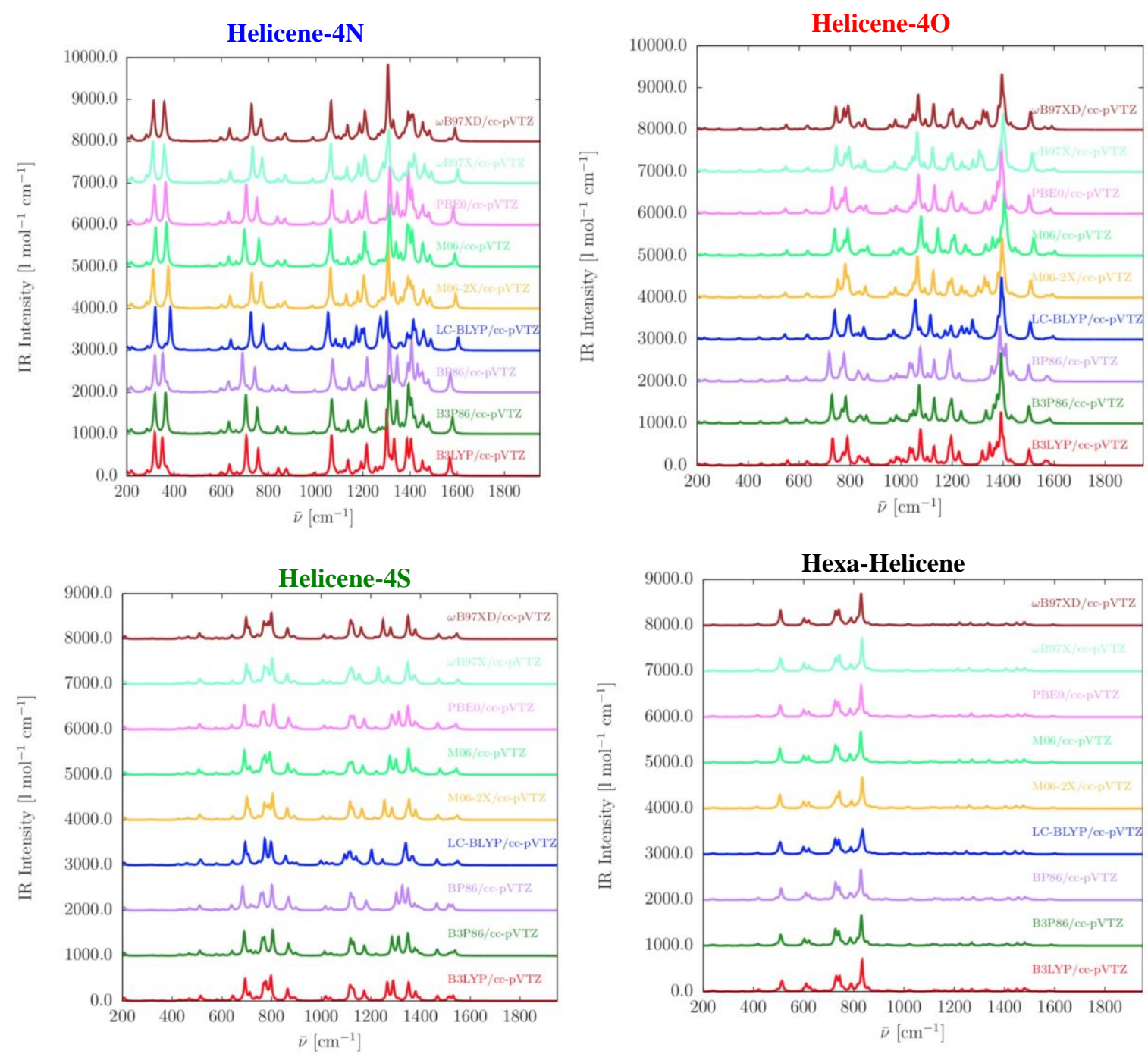

Figure 3. Simulated IR spectra of Helicene-4N, Helicene-4O, Helicene-4S, and Hexa-Helicene. The normal vibrational coordinates have been evaluated using various DFT functionals with the cc-pVTZ basis set, whereas the derivatives of the dipole moment at the CP-TDHF/aug-cc-pVDZ level. Each transition is represented by a Lorentzian function with FWHM of $10 \mathrm{~cm}^{-1}$

One can note that, for Helicene-4N, Helicene-4O, and Hexa-Helicene, functional overlaps are observed for the Raman spectra, which range from 0.890 to 0.995 for B3P86, BP86, PBE0, and $\omega$ B97X-D functionals.
For Helicene-4S, the overlaps of the Raman spectra give less good values, which amount to 0.831 at 0.878 with these functionals. Then, the values obtained for the overlaps of IR spectra have almost the same 
magnitudes as in the overlaps of the Raman spectra for Helicene-4O and Hexa-Helicene with B3LYP, BP86, and PBE0 functionals. The best correlation of the overlaps spectra with IR was obtained for B3P86, BP86, PBE0, and $\omega$ B97X-D functionals on Helicene$4 \mathrm{~N}$, which range from 0.819 to 0.840 . However, for Helicene-4S, the best values of the overlaps of the IR spectra were obtained with M06, M06-2X, and
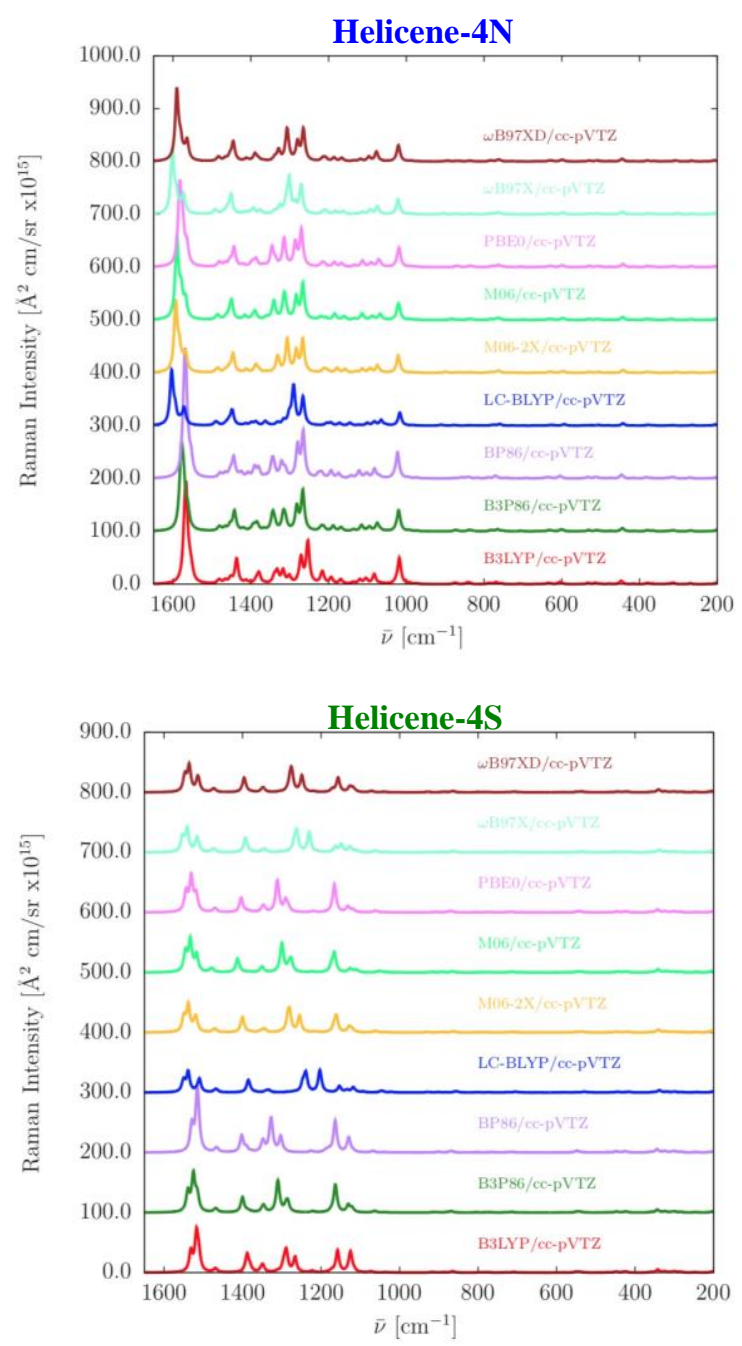

$\omega \mathrm{B} 97 \mathrm{X}$ and $\omega \mathrm{B} 97 \mathrm{X}-\mathrm{D}$ functionals.

For this purpose, we note at the end of these analyses that only two functionals, B3P86 and PBE0, give results that are very close to those of B3LYP functional. The simulated IR and Raman polarized spectra further evidence this in Figures 2 and 3.
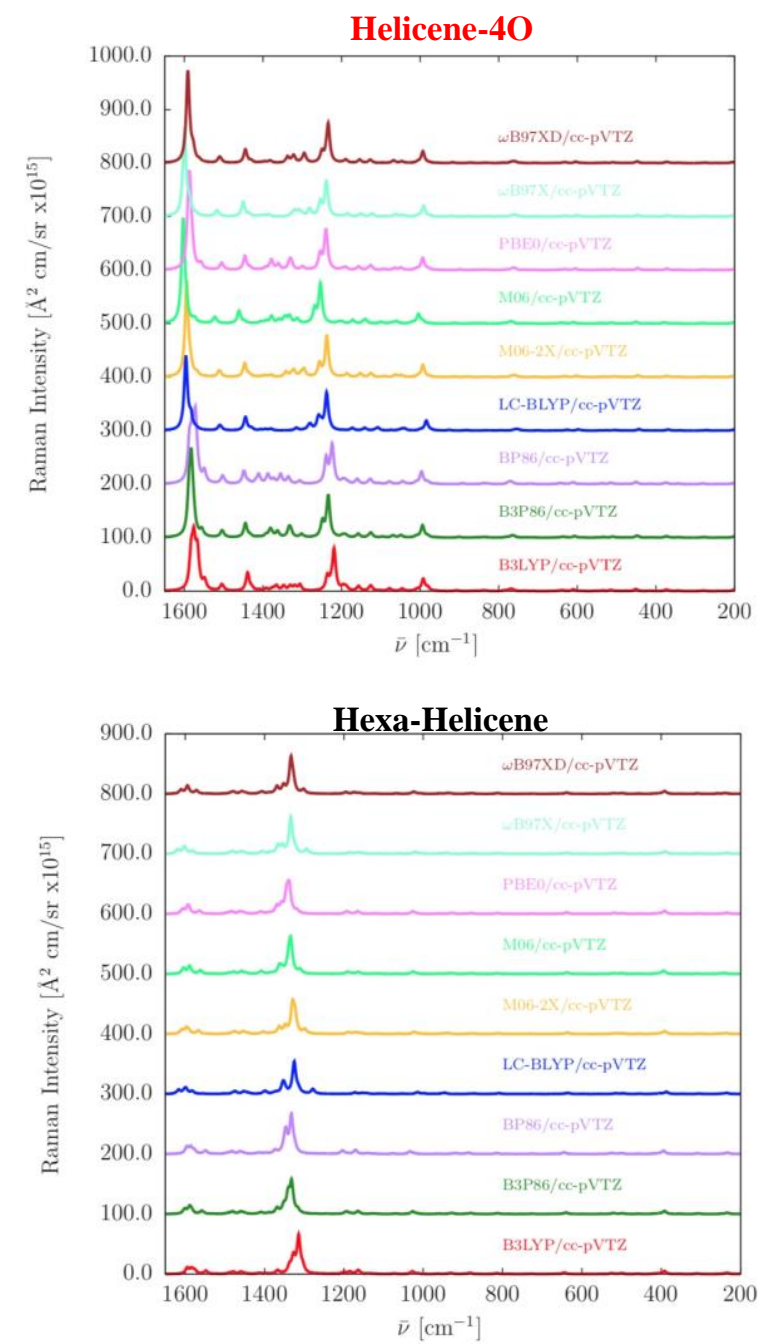

Figure 4. Simulated Raman spectra of Helicene-4N, Helicene-4O, Helicene-4S, and Hexa-Helicene. The normal vibrational coordinates have been evaluated using various DFT functionals with the cc-pVTZ basis set, whereas the derivatives of the polarizability at the CP-TDHF/aug-cc-pVDZ level with an optical wavelength of $532 \mathrm{~nm}$. Each transition is represented by a Lorentzian function with FWHM of $10 \mathrm{~cm}^{-1}$, which is less than obtained in the experimental spectra

\subsection{Comparison between calculated and experimental spectra}

The geometries optimized in B3LYP/cc-pVTZ calculations methods of 2-Br-Hexa-Helicene and 5-N-Hexa-Helicene showing the experimental IR and Raman spectra, which are juxtaposed and compared with, calculated one in the same quantum approach $^{38-41}$.

The vibrational signatures are compared to the experimental ones for the 2-Br-Hexa-Helicene ${ }^{13}$ and
5-N-Hexa-Helicene ${ }^{15}$ (Figure 5). The equilibrium geometries and the frequencies of the normal vibrational modes have been calculated with four XC functionals: B3LYP, B3P86, BP86, and PBE0 with cc-pVTZ basis set, whereas the properties derivative $\partial \mu / \partial \mathrm{R}$ and $\partial \alpha / \partial \mathrm{R}$ are evaluated with $\mathrm{CP}-\mathrm{TDHF} / \mathrm{aug}$ cc-pVDZ method. We observed that the four simulations (B3LYP, B3P86, BP86 and PBE0) reproduce the experimental data (Figures 6 and 7). 


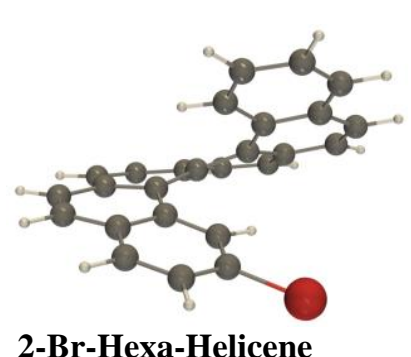

2-Br-Hexa-Helicene

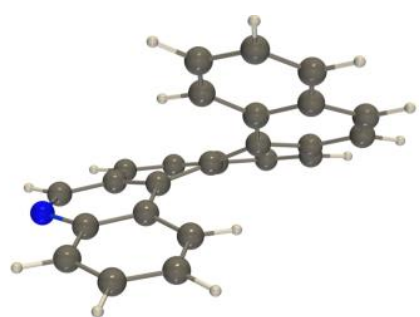

5-N-Hexa-Helicene

Figure 5. Sketch of the B3LYP/cc-pVTZ optimized structures of 2-Br-Hexa-Helicene and 5-N-Hexa- Helicene such as experimental IR and Raman spectra comparisons

However, slightly better results are obtained with B3LYP and B3P86 functionals.

The IR absorption spectra is dominated by three regions from 400 to $600 \mathrm{~cm}^{-1}, 800$ to $1000 \mathrm{~cm}^{-1}$, and 1400 to $1600 \mathrm{~cm}^{-1}$ with a very intense band at
$870 \mathrm{~cm}^{-1}$ together with a shoulder around $860 \mathrm{~cm}^{-1}$. All four simulations reproduce quite well the three regions. One should note that the missing signatures in the experimental spectrum at around $700 \mathrm{~cm}^{-1}$ and $1050 \mathrm{~cm}^{-1}$, which is due to the strong absorption of the solvent, which has been removed from the spectrum.

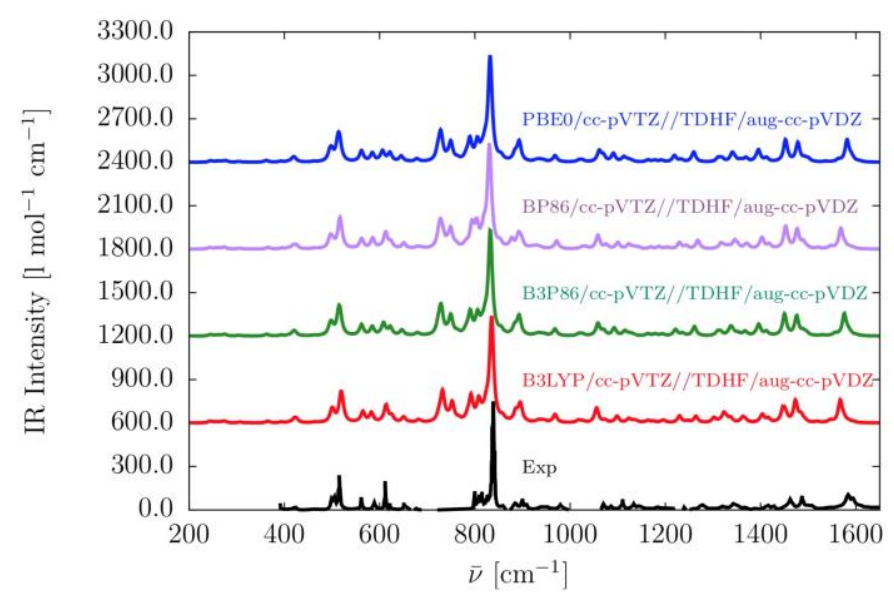

Figure 6. Experimental (Ref. ${ }^{13}$ ) and simulated IR spectra of 2-Br-Hexa-Helicene. The normal vibrational coordinates have been evaluated with B3LYP, B3P86, BP86 and PBE0 functionals with the cc-pVTZ basis set. In contrast, the derivatives of the dipole moment are using CP-TDHF/aug-cc-pVDZ level. Each transition is represented by a Lorentzian function with FWHM of $10 \mathrm{~cm}^{-1}$

The Raman polarized spectra are dominated by a band located between 1300 and $1400 \mathrm{~cm}^{-1}$. All four simulations reproduce quite well the experimental data ${ }^{13,15}$ even if slightly better results are obtained with B3LYP and B3P86 functionals.
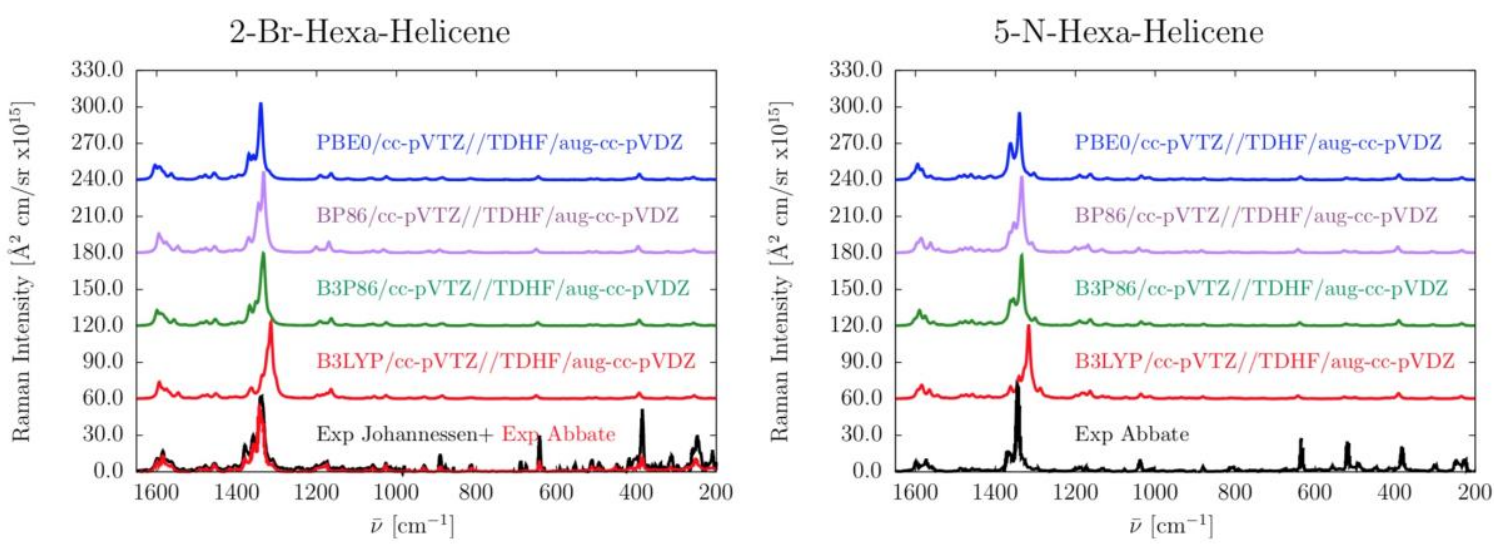

Figure 7. Experimental (Ref. ${ }^{13,15}$ ) and simulated Raman spectra of 2-Br-Hexa-Helicene (left) and 5-N-HexaHelicene (right). The normal vibrational coordinates have been evaluated with B3LYP, B3P86, BP86, and PBE0 functionals with the cc-pVTZ basis set, whereas the derivatives of the polarizability using CP-TDHF/aug-ccpVDZ level with an optical wavelength of $532 \mathrm{~nm}$. Each transition is represented by a Lorentzian function with

FWHM of $10 \mathrm{~cm}^{-1}$, and a multiplicative factor of 0.96 was used to scale the vibrational frequencies 
In the reception of the incident wave of $532 \mathrm{~nm}$ by Helicenes molecules, the normal vibrational modes, which generate the deformation of geometries, were observed in the frequency band of $800-1600 \mathrm{~cm}^{-1}$ (Fig.8). The effects of distortions of geometries, in the gaseous state and solution, cause a permutation of the diagonalized modes, and an inversion of the normal modes of vibrations. However, identical modes of vibration were noted in these two states, with a recovery rate higher than 0.950 .

The geometrical deformations are more observed on the functionalized Helicenes and less on the HexaHelicene. This shows that the functionalized Helicenes are more sensitive to visible radiation and will, therefore, be used in synthetic materials for light waveguides.

Some Vibrational Normal Modes of Helicene-4N

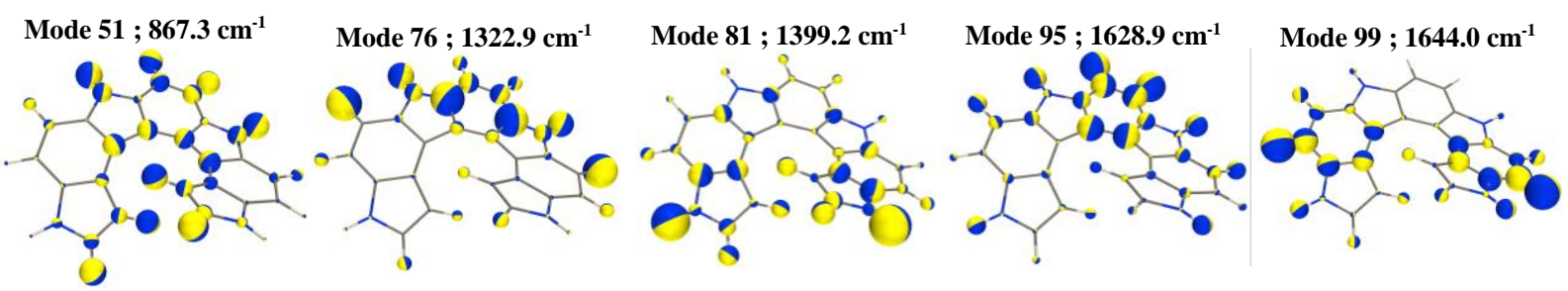

Some Vibrational Normal Modes of Helicene-4O

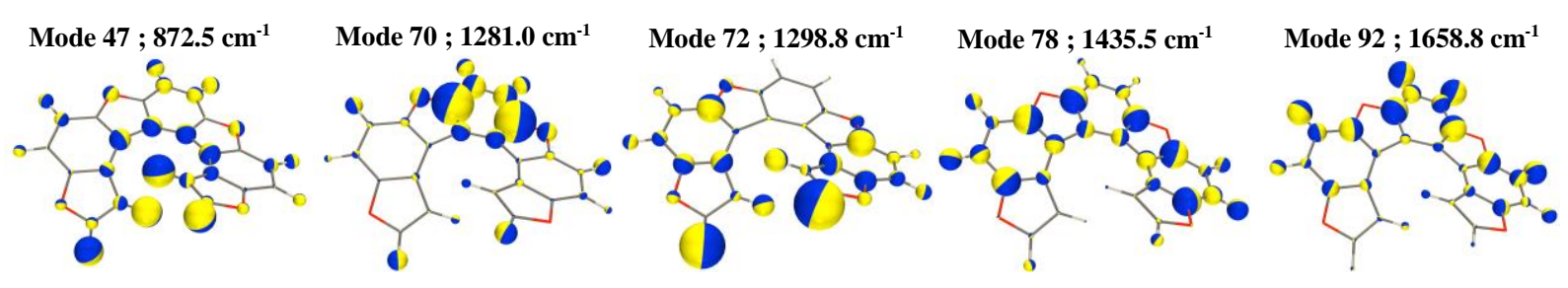

Some Vibrational Normal Modes of Helicene-4S

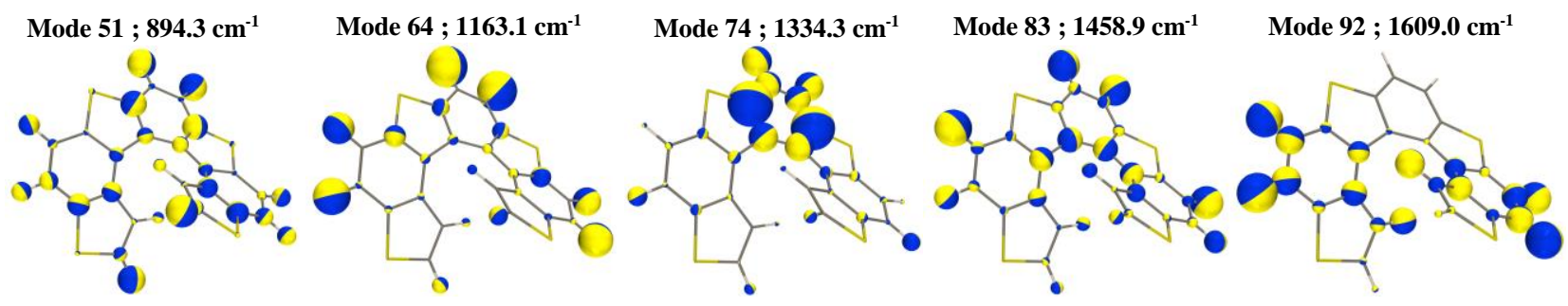

Some Vibrational Normal Modes of Hexa-Helicene
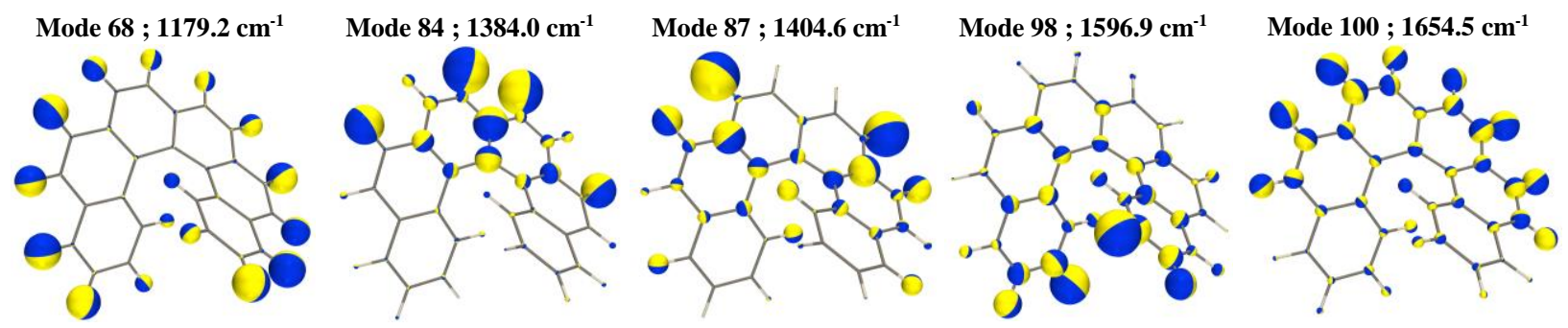

Figure 8. Sketch of the B3LYP/cc-pVTZ normal modes of vibration of Helicene-4N, Helicene-4O, Helicene-4S, and Hexa-Helicene molecules for the selected modes in the $800-1600 \mathrm{~cm}^{-1}$. The direction of atomic displacements is perpendicular to the junction plane between the two hemispheres of distinct color while their amplitudes are proportional to the radius of the sphere.

\section{Conclusions and outlook}

We have investigated the IR, and Raman signatures of four functionalized Helicenes and, in particular, the impact of the XC functional on their signatures. The simulation of the IR and Raman signatures involves two different steps, and therefore the impact of the method on both steps has been highlighted. The first step consists in the optimization of the geometry and the calculation of the normal vibrational modes. The 
second part is the evaluation of the Cartesian derivatives of the properties $(\partial \mu / \partial \mathrm{R}$ and $\partial \alpha / \partial \mathrm{R})$.

First, we have analyzed the impact of the $\mathrm{XC}$ functional on the normal modes and their influence on the IR and Raman signatures while keeping the method to calculate the properties derivatives of dipole moment and the polarizability $(\partial \mu / \partial \mathrm{R}$ and $\partial \alpha / \partial \mathrm{R})$ fixed (CP-TDHF/aug-cc-pVDZ). We have concluded that the choice of $\mathrm{XC}$ functional is more important and that like B3LYP, B3P86, and PBE0, reproduces well experimental spectra.

Second, starting from the B3LYP/cc-pVTZ structures and normal vibrational modes, the properties derivatives $(\partial \mu / \partial \mathrm{R}$ and $\partial \alpha / \partial \mathrm{R})$ have been evaluated with various $\mathrm{XC}$ functionals. The impact of the electron correlation was found to be rather limited. We conclude, therefore, that the CP-TDHF/rDPS: $3-21 \mathrm{G}$ level of theory is sufficient to provide specific properties derivatives $(\partial \mu / \partial \mathrm{R}$ and $\partial \alpha / \partial \mathrm{R})$. Overall, the most crucial point is to have an accurate description of the normal vibrational modes via the choice of an appropriate XC functional.

\section{Acknowledgments}

Simplice KOUDJINA, thanks to the Fund for the Research at the Laboratory of Theoretical Chemistry of the University of Namur and the PAI "P7/5 Functional Supramolecular Systems" for their support in the realization of this work under the direction of Professor. Benoît Champagne and Doctor. Vincent Liégeois. The calculations were performed on the computing facilities of the Consortium des Equipements de Calcul Intensif (CECI, http://www.ceci-hpc.be), particularly those of the Technological Platform on High-Performance Computing, for which we gratefully acknowledge the financial support of the FNRS-FNRC (Conventions 2.4.617.07.F and 2.5020.11) and the University of Namur.

\section{Conflict of Interest}

The authors declare that they have no conflict of interest.

\section{References}

1- R.H. Martin, The Helicenes, Angewandte Chemie International Edition in English, 1974, 13, 649-660.

2- C.F. Chen, Y. Shen, Helicene Chemistry, Springer Berlin Heidelberg: Berlin, Heidelberg, 2017.

3- J. Barroso, J.L. Cabellos, S. Pan, F. Murillo, X. Zarate, M.A. Fernandez-Herrera, G. Merino, Revisiting the racemization mechanism of helicenes, Chemical Communications, 2018, 54, 188-191.

4- J. Autschbach, S. Patchkovskii, T. Ziegler, S.J.A. van Gisbergen, E. Jan Baerends, Chiroptical properties from time-dependent density functional theory. II. Optical rotations of small to medium-sized organic molecules, The Journal of Chemical Physics, 2002, 117, 581-592.

5- H. Zhang, H. Liu, C. Shen, F. Gan, X. Su, H. Qiu, B. Yang, P. Yu, Chiral Recognition of Hexahelicene on a Surface via the Forming of Asymmetric Heterochiral Trimers, International Journal of Molecular Sciences, 2019, 20.

6- F. Furche, R. Ahlrichs, C. Wachsmann, E. Weber, A. Sobanski, F. Vögtle, S. Grimme, Circular Dichroism of Helicenes Investigated by Time-Dependent Density Functional Theory, Journal of the American Chemical Society, 2000, 122, 1717-1724.

7- J. Autschbach, T. Ziegler, S.J.A. van Gisbergen, E. J. Baerends, Chiroptical properties from timedependent density functional theory. I. Circular dichroism spectra of organic molecules, The Journal of Chemical Physics, 2002, 116, 6930-6940.

8- M. Spassova, I. Asselberghs, T. Verbiest, K. Clays, E. Botek, B. Champagne, Theoretical investigation on bridged triarylamine helicenes: UV/visible and circular dichroism spectra, Chemical Physics Letters, 2007, 439, 213-218.

9- E. Botek, B. Champagne, Circular dichroism of helical structures using semiempirical methods, The Journal of Chemical Physics, 2007, 127, 204101.

10-Y. Nakai, T. Mori, Y. Inoue, Theoretical and Experimental Studies on Circular Dichroism of Carbo[ $n$ ]helicenes, The Journal of Physical Chemistry A, 2012, 116, 7372-7385.

11-Y. Nakai, T. Mori, Y. Inoue, Circular Dichroism of (Di)methyl- and Diaza[6]helicenes.

A Combined Theoretical and Experimental Study, The Journal of Physical Chemistry A, 2013, 117, 83-93.

12-T. Bürgi, A. Urakawa, B. Behzadi, K.H. Ernst, A. Baiker, The absolute configuration of heptahelicene: aVCD spectroscopy study, New J Chem., 2004, 28, 332-334.

13-V.P. Nicu, J. Neugebauer, S.K. Wolff, E.J. Baerends, A vibrational circular dichroism implementation within a Slater-type-orbital based density functional framework and its application to Hexa- and hepta-helicenes, Theoretical Chemistry Accounts, 2008, 119, 245-263.

14-M. Kurban, Electronic structure, optical and structural properties of $\mathrm{Si}, \mathrm{Ni}, \mathrm{B}$, and $\mathrm{N}$-doped a carbon nanotube: DFT study, Optik, 2018, 172, 295-301.

15-S. Abbate, F. Lebon, G. Longhi, F. Fontana, T. Caronna, D.A. Lightner, Experimental and calculated vibrational and electronic circular dichroism spectra of 2-Br-hexahelicene, Physical Chemistry Chemical Physics, 2009, 11, 9039.

16-V. Liégeois, B. Champagne, Vibrational Raman optical activity of $\pi$-conjugated helical systems: Hexahelicene and heterohelicenes, Journal of Computational Chemistry, 2009, 30, 1261-1278. 
17-C. Johannessen, E.W. Blanch, C. Villani, S. Abbate, G. Longhi, N.R. Agarwal, M. Tommasini, D.A. Lightner, Raman and ROA Spectra of (-)- and (+)-2-Br-Hexahelicene: Experimental and DFT Studies of a $\pi$-Conjugated Chiral System, The Journal of Physical Chemistry B, 2013, 117, 2221-2230.

18-S. Abbate, G. Longhi, F. Lebon, E. Castiglioni, S. Superchi, L. Pisani, F. Fontana, F. Torricelli, T. Caronna, C. Villani, R. Sabia, M. Tommasini, A. Lucotti, D. Mendola, A. Mele, D.A. Lightner, Helical Sense-Responsive and SubstituentSensitive Features in Vibrational and Electronic Circular Dichroism, in Circularly Polarized Luminescence, and in Raman Spectra of Some Simple Optically Active Hexahelicenes, The Journal of Physical Chemistry C, 2014, 118, 1682-1695.

19-W.J. Hehre, R. Ditchfield, J.A. Pople, SelfConsistent Molecular Orbital Methods. XII. Further Extensions of Gaussian-Type Basis Sets for Use in Molecular Orbital Studies of Organic Molecules, The Journal of Chemical Physics, 1972, 56, 2257-2261.

20-T.H. Dunning, Gaussian basis sets for use in correlated molecular calculations. I. The atoms boron through neon and hydrogen, The Journal of Chemical Physics, 1989, 90, 1007-1023.

21-A. Komornicki, G. Fitzgerald, Molecular gradients, and hessians implemented in density functional theory, The Journal of Chemical Physics, 1993, 98, 1398-1421.

22-A.P. Scott, L. Radom, Harmonic Vibrational Frequencies: An Evaluation of Hartree-Fock, Møller-Plesset, Quadratic Configuration Interaction, Density Functional Theory, and Semiempirical Scale Factors, The Journal of Physical Chemistry, 1996, 100, 16502-16513.

23-K.K. Irikura, R.D. Johnson, R.N. Kacker, Uncertainties in Scaling Factors for ab Initio Vibrational Frequencies, The Journal of Physical Chemistry A, 2005, 109, 8430-8437.

24-M.P. Andersson, P. Uvdal, New Scale Factors for Harmonic Vibrational Frequencies Using the B3LYP Density Functional Method with the Triple- $\zeta$ Basis Set 6-311+G(d,p), The Journal of Physical Chemistry A, 2005, 109, 2937-2941.

25-G. Zuber, W. Hug, Rarefied Basis Sets for the Calculation of Optical Tensors. 1. The Importance of Gradients on Hydrogen Atoms for the Raman Scattering Tensor, The Journal of Physical Chemistry A, 2004, 108, 2108-2118.

26-A.J. Sadlej, Medium-size polarized basis sets for high-level-correlated calculations of molecular electric properties: II. Second-row atoms: $\mathrm{Si}$ through Cl, Theoretica Chimica Acta, 1991, 79, 123-140.

27-J.P. Merrick, D. Moran, L. Radom, An Evaluation of Harmonic Vibrational Frequency Scale Factors, The Journal of Physical Chemistry A, 2007, 111, 11683-11700.

28-M.J. Frisch, G.W. Trucks, H.B. Schlegel, G.E.
Scuseria, M.A. Robb, J. R. Cheeseman, G. Scalmani, V. Barone, B. Mennucci, G.A. Petersson, H. Nakatsuji, M. Caricato, X. Li, H.P. Hratchian, A.F. Izmaylov, J. Bloino, G. Zheng, J.L. Sonnenberg, M. Hada, M. Ehara, K. Toyota, R. Fukuda, J. Hasegawa, M. Ishida, T. Nakajima, Y. Honda, O. Kitao, H. Nakai, T. Vreven, J.A. Montgomery, J.E. Peralta, F. Ogliaro, M. Bearpark, J.J. Heyd, E. Brothers, K.N. Kudin, V.N. Staroverov, T. Keith, R. Kobayashi, J. Normand, K. Raghavachari, A. Rendell, J.C. Burant, S.S. Iyengar, J. Tomasi, M. Cossi, N. Rega, J.M. Millam, M. Klene, J.E. Knox, J.B. Cross, V. Bakken, C. Adamo, J. Jaramillo, R. Gomperts, R.E. Stratmann, O. Yazyev, A.J. Austin, R. Cammi, C. Pomelli, J.W. Ochterski, R.L. Martin, K. Morokuma, V.G. Zakrzewski, G.A. Voth, P. Salvador, J.J. Dannenberg, S. Dapprich, A.D. Daniels, O. Farkas, J.B. Foresman, J.V. Ortiz, J. Cioslowski, D.J. Fox, Gaussian, Inc., Wallingford CT, Gaussian 09, Revision D.01, 2013.

29-E. Lamparska, V. Liégeois, O. Quinet, B. Champagne, Theoretical Determination of the Vibrational Raman Optical Activity Signatures of Helical Polypropylene Chains, ChemPhysChem, 2006, 7, 2366-2376.

30-V. Liégeois, B. Champagne, Implementation in the Pyvib2 program of the localized mode method and application to a helicene, Theoretical Chemistry Accounts, 2012, 131.

31-V. Liégeois, O. Quinet, B. Champagne, Investigation of polyethylene helical conformations: Theoretical study by vibrational Raman optical activity, International Journal of Quantum Chemistry, 2006, 106, 3097-3107.

32-V. Liégeois, O. Quinet, B. Champagne, Vibrational Raman optical activity as a mean for revealing the helicity of oligosilanes: A quantum chemical investigation, The Journal of Chemical Physics, 2005, 122, 214304.

33-L.D. Barron, A.D. Buckingham, Rayleigh, and Raman scattering from optically active molecules, Molecular Physics, 1971, 20, 1111-1119.

34-L.D. Barron, Molecular Light Scattering and Optical Activity, 2nd ed. Cambridge University Press, 2004.

35-F. Cailliez, P. Pernot, Statistical approaches to forcefield calibration and prediction uncertainty in molecular simulation, The Journal of Chemical Physics, 2011, 134, 054124.

36-R.L. Jacobsen, R.D. Johnson, K.K. Irikura, R.N. Kacker, Anharmonic Vibrational Frequency Calculations Are Not Worthwhile for Small Basis Sets, Journal of Chemical Theory and Computation, 2013, 9, 951-954.

37-A.D. Buckingham, Permanent and Induced Molecular Moments and Long-Range Intermolecular Forces, Advances in Chemical Physics: Intermolecular Forces, 2007, 107-142. 38-C. Baldoli, A. Bossi, C. Giannini, E. Licandro, 
S. Maiorana, D. Perdicchia, M. Schiavo, A Novel and Efficient Approach to (Z)-1,2-

Bis(benzodithienyl)ethene -Precursors of

Tetrathia[7]helicenes, Synlett, 2005, 1137-1141.

39-J. Žádný, P. Velíšek, M. Jakubec, J. Sýkora,

V. Církva, J. Storch, Exploration of

9-bromo[7]helicene reactivity, Tetrahedron, 2013, 69, 6213-6218.
40- E. Licandro, S. Cauteruccio, D. Dova,

Thiahelicenes, Advances in Heterocyclic Chemistry, 2016, 118, 1-46.

41-A. Bossi, S. Maiorana, C. Graiff, A. Tiripicchio,

E. Licandro, Silyl-Substituted

Tetrathia[7]helicenes: Synthesis, X-ray

Characterization, and Reactivity, European

Journal of Organic Chemistry, 2007, 4499-4509. 